\title{
Structural relaxation in Morse clusters: Energy landscapes
}

\author{
Mark A. Miller, Jonathan P. K. Doye, and David J. Wales \\ University Chemical Laboratory, Lensfield Road, Cambridge CB2 1EW, United Kingdom
}

(Received 10 August 1998; accepted 29 September 1998)

\begin{abstract}
We perform a comprehensive survey of the potential energy landscapes of 13-atom Morse clusters, and describe how they can be characterized and visualized. Our aim is to detail how the global features of the funnel-like surface change with the range of the potential, and to relate these changes to the dynamics of structural relaxation. We find that the landscape becomes rougher and less steep as the range of the potential decreases, and that relaxation paths to the global minimum become more complex. (C) 1999 American Institute of Physics. [S0021-9606(99)03001-9]
\end{abstract}

\section{INTRODUCTION}

Structural relaxation plays a key role in a diverse range of problems in chemical physics, including protein folding, glass formation, and the observation of "magic number" peaks in the mass spectrometry of rare gas clusters. The dynamic evolution of such systems is determined by the potential energy surface (PES) generated by the interactions between their constituent particles. Quite often one wants to find the structure and physical properties of a (macro)molecule or cluster, by which it is usually meant the properties of the global minimum on the PES, or, equivalently, the properties at zero Kelvin. However, the dynamics of a system at temperatures or energies above which it can escape from the global minimum depend on larger regions of the PES, the topology, and topography of which determine the precise behavior. When considering the wider features of the PES in this way, it has become usual to refer to the PES as the "potential energy landscape."

One can also consider the free energy landscape, a temperature-dependent function which incorporates the entropy. For example, in protein folding such a landscape can be defined either as a function of the protein configuration by averaging the free energy over all solvent coordinates, or as a function of distance from the folded state in terms of a similarity parameter. ${ }^{1}$

In recent years, much understanding has been gained in a number of fields by relating structural and dynamical properties to the underlying PES. For example, many years ago Levinthal pointed out the apparent contradiction between the astronomical number of possible configurations that a protein can adopt and the rapidity with which it finds the biologically active structure when it folds. ${ }^{2,3}$ The "paradox" is resolved by realizing that efficient folding is only possible when the potential energy landscape is dominated by a funnel, i.e., consists largely of convergent kinetic pathways leading down in energy towards the required structure. ${ }^{4}$ The precise features of a funnel may vary, but the native state must be thermodynamically stable at temperatures or energies where the dynamics are fast enough for the system to be able to explore the landscape and find it. ${ }^{5}$ The native state is destabilized if there are structurally distinct states of low energy which can act as kinetic traps. ${ }^{6}$ Hence, a pronounced global minimum encourages efficient folding. ${ }^{7,8}$

The potential energy landscape also plays an important role in determining the behavior of bulk liquids. Angell has proposed a widely used scheme in which liquids are classified from "strong" to "fragile.", A strong liquid is characterized by a viscosity whose temperature dependence follows an Arrhenius relationship $(\propto \exp [A / T])$. These are often liquids with open network structures like water and $\mathrm{SiO}_{2}$, whereas fragile liquids tend to have more isotropic interactions. Angell ${ }^{10}$ and Stillinger ${ }^{11}$ have described the general features of the energy landscapes that might be expected to characterize the two extremes. In a recent study, Sastry et al. have investigated the role of different regions of the landscape in the process of glass formation in a model fragile liquid. ${ }^{12}$ They find that as the temperature of the liquid is decreased, the system samples regions with higher barriers, and on further cooling it samples deeper minima and nonexponential relaxation sets in.

Another way that an energy landscape can be classified is as "sawtooth-like" or "staircase-like" depending on the energy difference between minima relative to the barriers which separate them. ${ }^{13,14}$ For example, the "structureseeking" properties of the $(\mathrm{KCl})_{32}$ cluster (i.e., its ability to find a rock salt structure even when cooled rapidly) can be attributed to downhill barriers which are low compared to the potential energy gradient towards crystalline minima, as in a staircase.

In order to characterize an energy landscape, it is necessary to make a survey of its important features: minima, transition states and pathways. Since the number of such features increases at least exponentially with the number of particles in the system, ${ }^{15}$ it is impractical and undesirable to catalogue them all for large systems. Consequently, existing studies have usually concentrated on analyzing what is hoped to be a representative sample of minima and transition states. ${ }^{13,16}$ In this study we examine in detail the landscape of the 13-atom Morse cluster $\left(\mathrm{M}_{13}\right)$, which is large enough to possess a complex PES, but is small enough for us to make a nearly exhaustive list of its minima and transition states. This model system is especially interesting because the energy landscape is dominated by a funnel, and the potential contains one parameter which allows us to adjust the complexity of the PES. Previous studies ${ }^{17,18}$ have shown that potential 
energy surfaces are simpler for long-ranged potentials, and the effects of the range on the morphology of global minima of atomic clusters ${ }^{19,20}$ and the stability of simple liquids ${ }^{16,21}$ have already received attention. The range of the potential also affects phase behavior: in a study of 7-atom Morse clusters, Mainz and Berry found that liquid-like and solid-like phase coexistence is less distinct when the range of attraction is longer. ${ }^{22}$

In this paper, we concentrate on finding useful ways to characterize and visualize a complex PES, and in the Summary we comment on how the range of the potential is likely to affect the relaxation properties of the cluster. We are currently using the data collected in this study to perform master equation dynamics on the system to address relaxation in detail.

\section{EXPLORING THE LANDSCAPE}

The Morse potential ${ }^{23}$ can be written in the form

$$
V=\sum_{i<j} V_{i j} ; \quad V_{i j}=e^{\rho\left(1-r_{i j} / r_{\mathrm{e}}\right)}\left[e^{\rho\left(1-r_{i j} / r_{\mathrm{e}}\right)}-2\right] \epsilon,
$$

where $r_{i j}$ is the distance between atoms $i$ and $j . \epsilon$ and $r_{\mathrm{e}}$ are the dimer well depth and equilibrium bond length, and simply scale the PES without affecting its topology. They can conveniently be set to unity and used as the units of energy and distance. $\rho$ is a dimensionless parameter which determines the range of the interparticle forces, with low values corresponding to long range. Physically meaningful values range at least from $\rho=3.15$ and 3.17 for sodium and potassium ${ }^{24}$ to 13.62 for $\mathrm{C}_{60}$ molecules. ${ }^{25}$ When $\rho=6$, the Morse potential has the same curvature as the Lennard-Jones potential at the minimum.

The first step in characterizing the PES is to map out the local minima and the network of transition states ${ }^{26}$ and pathways that connects them. The eigenvector-following technique $^{27-29}$ can efficiently locate transition states (firstorder saddles) by maximizing the energy along a specified direction, while simultaneously minimizing in all other directions. The minima connected to a given transition state are defined by the steepest descent paths commencing parallel and antiparallel to the transition vector (the Hessian eigenvector with negative eigenvalue) at the transition state. Although eigenvector following can also be used for these minimizations, the pathways are not necessarily the same, ${ }^{29}$ and may even lead to a different minimum. Since both the pathways and the connectivity are of interest here, we use a steepest descent technique for minimizations, employing analytic second derivatives, following Page and McIver. ${ }^{30}$

Our algorithm for exploring the PES is similar to that used by Tsai and Jordan in a study of small Lennard-Jones and water clusters. ${ }^{31}$ Starting from a known minimum:

(1) Search for a transition state along the eigenvector with the lowest eigenvalue.

(2) Deduce the path through this transition state and the minima connected to it.

(3) Repeat from step 1 beginning antiparallel to the eigenvector, and then in both directions along eigenvectors
TABLE I. Details of the databases for $\mathrm{M}_{13}$ at four values of the range parameter $\rho . n_{\mathrm{ev}}$ is the minimum number of eigenvectors of each minimum searched for a transition state, and $n_{\mathrm{s}}$ is the average number of searches from each minimum. $n_{\min }$ and $n_{\mathrm{ts}}$ are the numbers of minima and transition states found.

\begin{tabular}{ccccc}
\hline \hline$\rho$ & 4 & 6 & 10 & 14 \\
\hline$n_{\mathrm{ev}}$ & 15 & 6 & 3 & 2 \\
$n_{\mathrm{s}}$ & 31.3 & 13.0 & 7.0 & 7.5 \\
$n_{\min }$ & 159 & 1439 & 9306 & 12760 \\
$n_{\mathrm{ts}}$ & 685 & 8376 & 37499 & 54439 \\
\hline \hline
\end{tabular}

with successively higher eigenvalues until a specified number, $n_{\mathrm{ev}}$, of directions have been searched uphill.

(4) Repeat from step 1 until $n_{\mathrm{ev}}$ modes of all known minima have been searched.

By taking steps directly between minima, this method avoids wasting time on intrawell dynamics. Other methods for exploring energy landscapes, such as molecular dynamics, can become trapped in local minima, especially at low temperature, where there is a wide separation in time scale between interwell and intrawell motion. The chosen value of $n_{\mathrm{ev}}$ clearly affects the thoroughness of the survey, although even if all $(3 N-6)$ vibrational modes of an $N$-atom cluster are searched, there is no guarantee of finding every minimum and transition state. In practice, the required computer time and storage demand that $n_{\mathrm{ev}}$ be reduced for large $\rho$, since the complexity of the PES increases dramatically as the range of the potential decreases. However, one finds that searches from low-lying minima are more likely to converge in a reasonable number of iterations, so the above algorithm was augmented with searches along further eigenvectors of lower-energy minima. We are confident that the databases generated for $\rho=4$ and 6 are nearly exhaustive, and although those for higher values of $\rho$ are necessarily less complete, this approach still allows us to map out the PES fairly comprehensively.

Details of the searches and the resulting databases for $\rho=4,6,10$, and 14 are summarized in Table I. The dramatic rise in the number of minima and transition states found as the range of the potential decreases is the first indication of the increasing complexity of the PES. ${ }^{17}$ The remainder of this paper investigates in more detail the nature of these changes and some useful ways of characterizing the landscapes.

\section{TOPOLOGICAL MAPPING}

When trying to describe an energy "landscape," one has already been forced to use terminology appropriate to a surface in three-dimensional space, and pictorial representations are usually restricted even further to two dimensions. Visualizing a $3 N$-dimensional object directly in such a way has obvious limitations, yet it is appealing to have an idea of "what the surface looks like."

One helpful way of doing this is to use topological mapping to construct a disconnectivity graph, as applied to a polypeptide by Becker and Karplus. ${ }^{32}$ The analysis begins by mapping every point in configuration space onto the local 
minimum reached by following the steepest descent path. ${ }^{15}$ Thus, configuration space is represented by the discrete set of minima, each of which has an associated "well' of points which map onto it. Although this approach discards information about the volume of phase space associated with each minimum, the density of minima can provide a qualitative impression of the volumes associated with the various regions of the landscape.

At a given total energy, $E$, the minima can be grouped into disjoint sets, called basins ("super basins" in Becker and Karplus' nomenclature), whose members are mutually accessible at that energy. In other words, each pair of minima in a basin are connected directly or through other minima by a path whose energy never exceeds $E$, but would require more energy to reach a minimum in another basin. At low energy there is just one basin-that containing the global minimum. At successively higher energies, more basins come into play as new minima are reached. At still higher energies, the basins coalesce as higher barriers are overcome, until finally there is just one basin containing all the minima (provided there are no infinite barriers).

The disconnectivity graph is constructed by performing the basin analysis at a series of energies, plotted on a vertical scale. At each energy, a basin is represented by a node, with lines joining nodes in one level to their daughter nodes in the level below. The choice of the energy levels is important; too wide a spacing and no topological information is left, whilst too close a spacing produces a vertex for every transition state and hides the longer range structure of the landscape. The horizontal position of the nodes is arbitrary, and can be chosen for clarity. In the resulting graph, all branches terminate at local minima, while all minima connected directly or indirectly to a node are mutually accessible at the corresponding energy.

The disconnectivity graphs for $\mathrm{M}_{13}$ with $\rho=4$ and 6 are plotted on the same scale in Fig. 1. We have chosen a linear energy spacing of one dimer well depth, which is an effective compromise between the points raised above. Both trees are typical of a funnel-like landscape: as the energy is lowered, minima are cut off a few at a time with no secondary funnels, which would appear as side branches. A large upward shift in the energy range of the minima is apparent on increasing $\rho$ from 4 to 6 , due to the increase in the energetic penalty for strain and a decrease in the energetic contribution from next-nearest neighbors as the range of the potential decreases. ${ }^{19}$ An increase in barrier heights is also revealed by the somewhat longer branches at $\rho=6$. Because of the large number of minima involved in the databases for $\rho=10$ and 14 , the disconnectivity graphs are too dense to illustrate, but we shall see in the numerical analysis of the next section how the trends develop.

The concepts involved in the disconnectivity graph have much in common with the "energy lid" description of Sibani et al. $^{33}$ in which minima are grouped together if they are connected by paths never exceeding a particular energy (the "lid"). These authors plotted a tree with a time axis, on which nodes represent the time when groups of minima first come into equilibrium.

The term "basin'" has been used with a somewhat dif-

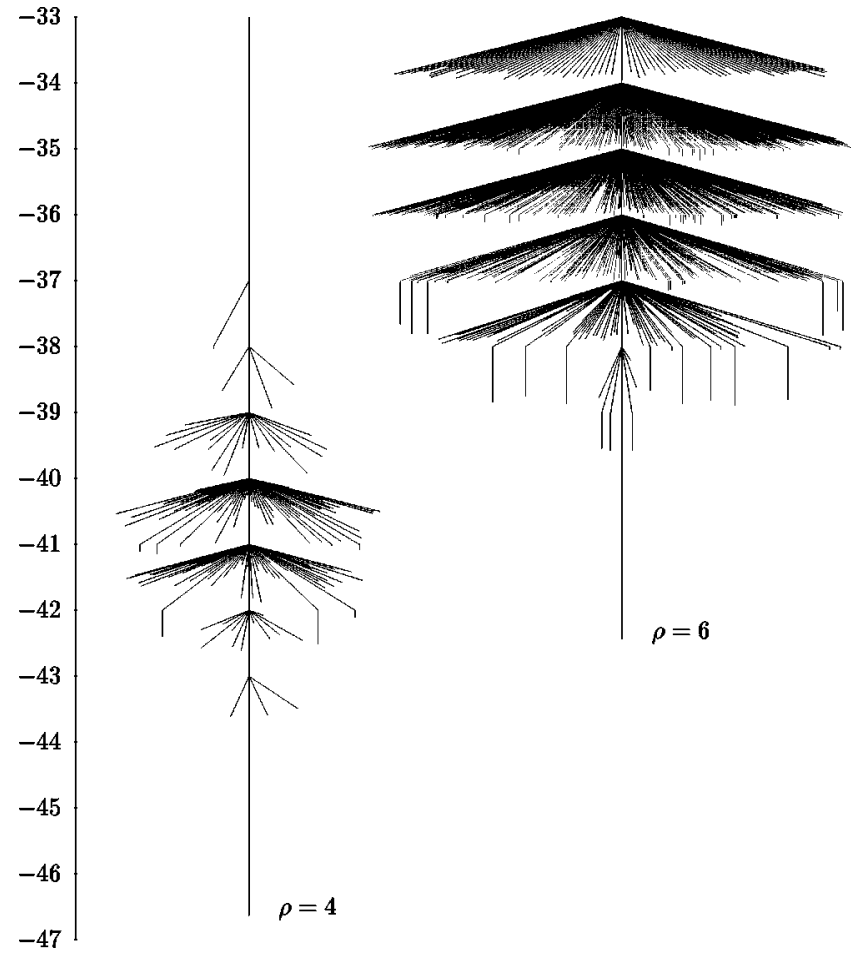

FIG. 1. Disconnectivity trees for $\mathrm{M}_{13}$ with $\rho=4$ and $\rho=6$ plotted on the same energy scale (in units of the pair well depth).

ferent meaning by Berry and co-workers. ${ }^{13,34}$ In this definition, a basin consists of all minima connected to the basin bottom by a monotonic sequence, i.e. a sequence of connected minima with monotonically decreasing energy. This definition contrasts with that of Becker and Karplus, ${ }^{32}$ because it is independent of the energy, and actually has a lot in common with the notion of a funnel. Although the word "funnel" may conjure up a misleading image when the surface is rough or shallow in slope, we will use it in this context to avoid confusion with the previous definition of a basin as a set of mutually accessible minima at a given energy. The funnel terminating at the global minimum is denoted the primary funnel, whilst adjoining side funnels are termed secondary. It should be noted that this definition permits a minimum to belong to more than one funnel via different transition states. The significance of dividing the landscape in this way is that interfunnel motion is likely to occur on a slower time scale than interwell flow, ${ }^{13,34}$ so funnels constitute the next level in a hierarchy of landscape structure. Sufficiently deep or voluminous secondary wells can act as traps. ${ }^{4,6} \mathrm{~A}$ striking example is the cluster of 38 Lennard-Jones atoms, whose truncated octahedral global minimum was only found quite recently ${ }^{19,35}$ because of the much larger secondary funnel associated with a low-lying icosahedral structure. ${ }^{36}$

As the first line of Table II shows, for $\rho=4$ the landscape of $\mathrm{M}_{13}$ is a perfect funnel: all minima lie on monotonic sequences terminating at the global minimum. At higher values of $\rho$ a small fraction of minima lie outside the primary funnel, and although they technically constitute secondary funnels, they represent a very small proportion of the phase space. We will now see how the characteristics of the primary funnel evolve as the range of the potential is decreased. 
TABLE II. Some properties of the potential energy landscape of $\mathrm{M}_{13}$ at four values of the range parameter $\rho$. All dimensioned quantities are tabulated in reduced units. $n_{\min }$ is the number of minima, of which $n_{\mathrm{pf}}$ lie in the primary funnel. $E_{\mathrm{gm}}$ is the energy of the global minimum, with the next-lowest energy structure lying $\Delta E_{\text {gap }}$ higher. $\bar{\nu}_{i}$ is the geometric mean normal mode frequency at minimum $i$ and $\nu_{i}^{\text {im }}$ is the imaginary frequency at transition state $i . b_{i}^{\text {up }}$ is the larger (uphill) barrier height between the two minima connected by transition state $i, b_{i}^{\text {down }}$ is the smaller (downhill) barrier, and $\Delta E_{i}^{\text {con }}$ is the energy difference between the minima, so that $b_{i}^{\text {up }}=b_{i}^{\text {down }}$ $+\Delta E_{i}^{\text {con }} . S_{i}$ is the integrated path length between the two minima connected by transition state $i, D_{i}$ is their separation in configuration space, and $\widetilde{N}_{i}$ is the cooperativity index of the rearrangement (defined in the text). $n_{i}^{\mathrm{gm}}$ is the smallest number of steps from minimum $i$ to the global minimum, and $S_{i}^{\text {gm }}$ is the integrated length of this path. $\langle\cdots\rangle_{\mathrm{m}},\langle\cdots\rangle_{\mathrm{ts}}$ and $\langle\cdots\rangle_{\mathrm{p}}$ indicate averages where the index runs over minima, transition states, and nondegenerate pathways (i.e., pathways not merely connecting permutational isomers), respectively.

\begin{tabular}{lcccc}
\hline \multicolumn{1}{c}{$\rho$} & 4 & 6 & 10 & 14 \\
\hline$n_{\min }-n_{\mathrm{pf}}$ & 0 & 1 & 219 & 442 \\
$E_{\mathrm{gm}}$ & -46.635 & -42.440 & -39.663 & -37.259 \\
$\Delta E_{\mathrm{gap}}$ & 3.024 & 2.864 & 2.245 & 0.468 \\
$\langle\bar{\nu}\rangle_{\mathrm{m}}$ & 1.187 & 1.625 & 2.615 & 3.660 \\
$\left\langle\mid \nu^{\text {im }}\right\rangle_{\mathrm{ts}}$ & 0.396 & 0.473 & 0.637 & 0.628 \\
$\left\langle b^{\mathrm{up}}\right\rangle_{\mathrm{p}}$ & 3.666 & 2.070 & 1.470 & 1.536 \\
$\left\langle b^{\text {down }}\right\rangle_{\mathrm{p}}$ & 0.461 & 0.543 & 0.583 & 0.784 \\
$\left\langle\Delta E^{\mathrm{con}}\right\rangle_{\mathrm{p}}$ & 3.205 & 1.526 & 0.887 & 0.752 \\
$\langle S\rangle_{\mathrm{p}}$ & 2.471 & 1.735 & 1.030 & 0.971 \\
$\langle D\rangle_{\mathrm{p}}$ & 1.469 & 1.163 & 0.840 & 0.817 \\
$\langle\tilde{N}\rangle_{\mathrm{p}}$ & 6.673 & 5.939 & 6.093 & 5.918 \\
$\left\langle n^{\mathrm{gm}}\right\rangle_{\mathrm{m}}$ & 1.354 & 2.447 & 3.744 & 3.885 \\
$\left\langle S^{\mathrm{gm}}\right\rangle_{\mathrm{m}}$ & 2.772 & 3.534 & 3.573 & 3.357 \\
\hline \hline
\end{tabular}

\section{PROPERTIES OF THE LANDSCAPE}

The remainder of Table II lists some global properties of the landscape at four values of $\rho$. Some of the trends are straightforward to understand. For example, defining $\bar{\nu}_{i}$ as the geometric mean of the normal mode frequencies at minimum $i$, the average of this quantity over the database of minima, $\langle\bar{\nu}\rangle_{\mathrm{m}}$, rises monotonically with $\rho$ because of the increasing stiffness of shorter-ranged potentials. The average of the transition state imaginary frequency, $\nu_{i}^{\text {im }}$, increases less rapidly in magnitude, and levels off at high $\rho$, indicating that the transition regions are flatter relative to the well bottoms than at low $\rho$.

The increasing energy, $E_{\mathrm{gm}}$, of the global minimum was noted in the previous section, and the table shows that this increase is accompanied by a decreasing gap $\Delta E_{\text {gap }}$ to the second lowest minimum. The striking drop in $\Delta E_{\text {gap }}$ when $\rho$ reaches 14 is due to a change in morphology of the second lowest structure, as illustrated in Fig. 2. To see why this happens, it is helpful to decompose the potential energy into the following contributions: ${ }^{37}$

$$
V=-n_{\mathrm{nn}}+E_{\text {strain }}+E_{\mathrm{nnn}},
$$

where $n_{\mathrm{nn}}$ is the number of nearest-neighbor interactions, i.e., the number of pairs lying closer than a value $r_{0}$ (taken here to be $1.15 r_{\mathrm{e}}$ ), and the strain energy and non-nearestneighbor contributions are defined by

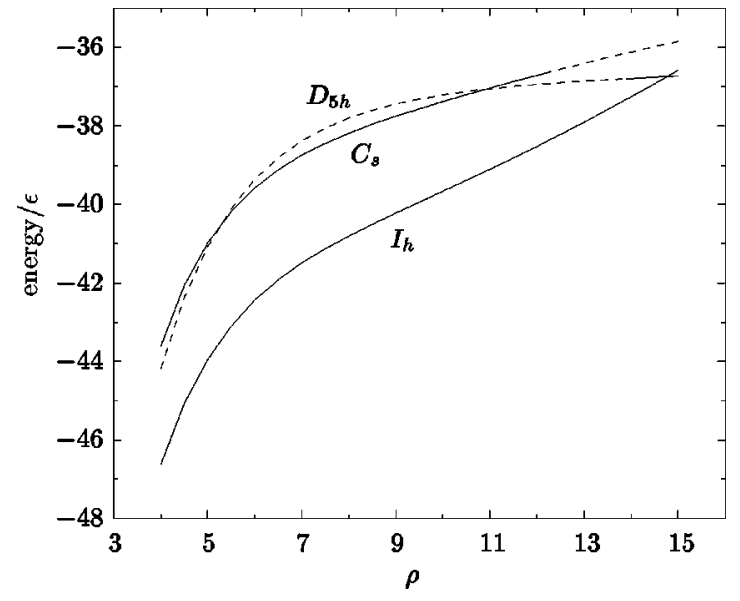

FIG. 2. Correlation diagram for some low-lying structures (see Fig. 3): the icosahedron $\left(I_{h}\right)$, the decahedron $\left(D_{5 h}\right)$ and the lowest-energy defective icosahedron $\left(C_{s}\right)$. Dashed lines indicate regions where the structure is not a minimum: $D_{5 h}$ becomes a transition state and $C_{s}$ becomes a second-order saddle.

$$
\begin{aligned}
& E_{\text {strain }}=\sum_{\substack{i<j \\
r_{i j}<r_{0}}}\left[V_{i j}+1\right], \\
& E_{\mathrm{nnn}}=\sum_{\substack{i<j \\
r_{i j} \geqslant r_{0}}} V_{i j} .
\end{aligned}
$$

$n_{\mathrm{nn}}$ and $E_{\text {strain }}$ are more sensitive properties of the structure than $E_{\text {nnn }}$, and so the lowest energy cluster is determined by a balance between maximizing $n_{\mathrm{nn}}$ and minimizing $E_{\text {strain }}$. The icosahedron [Fig. 3(a)] is the global minimum for all four values of $\rho$ considered here because it has the largest number of nearest neighbors $\left(n_{\mathrm{nn}}=42\right)$. However, the large value of $n_{\mathrm{nn}}$ is at the expense of considerable strain. As $E_{\text {strain }}$ is the energetic penalty for nearest-neighbor distances deviating from $r_{\mathrm{e}}$, it increases rapidly for strained structures as the pair-potential well narrows at larger $\rho . E_{\mathrm{nnn}}$ is also sensitive to $\rho$; it decreases as the range of the potential decreases.

The upward trends in Fig. 2 are caused by the changes in $E_{\text {strain }}$ and $E_{\text {nnn }}$. For $\rho<13.90$, the second lowest minimum is a defective icosahedron in which one vertex has been removed and one face is capped [Fig. 3(b)]. The removal of a vertex allows the strain in the icosahedron to relax, and so the energy rises less steeply than for the icosahedron and $\Delta E_{\text {gap }}$ falls. However, decahedral clusters are intrinsically less strained than icosahedral ones, and at $\rho=13.90$ the decahedron [Fig. 3(c)], which for lower $\rho$ is a transition state,

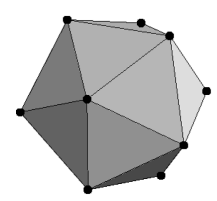

(a)

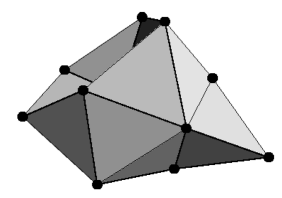

(b)

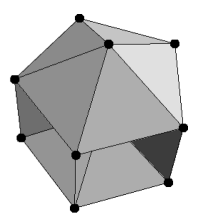

(c)
FIG. 3. Structures discussed in the text: (a) the icosahedron $\left(I_{h}\right)$, (b) the lowest-energy defective icosahedron $\left(C_{s}\right)$, and (c) the decahedron $\left(D_{5 h}\right)$. 

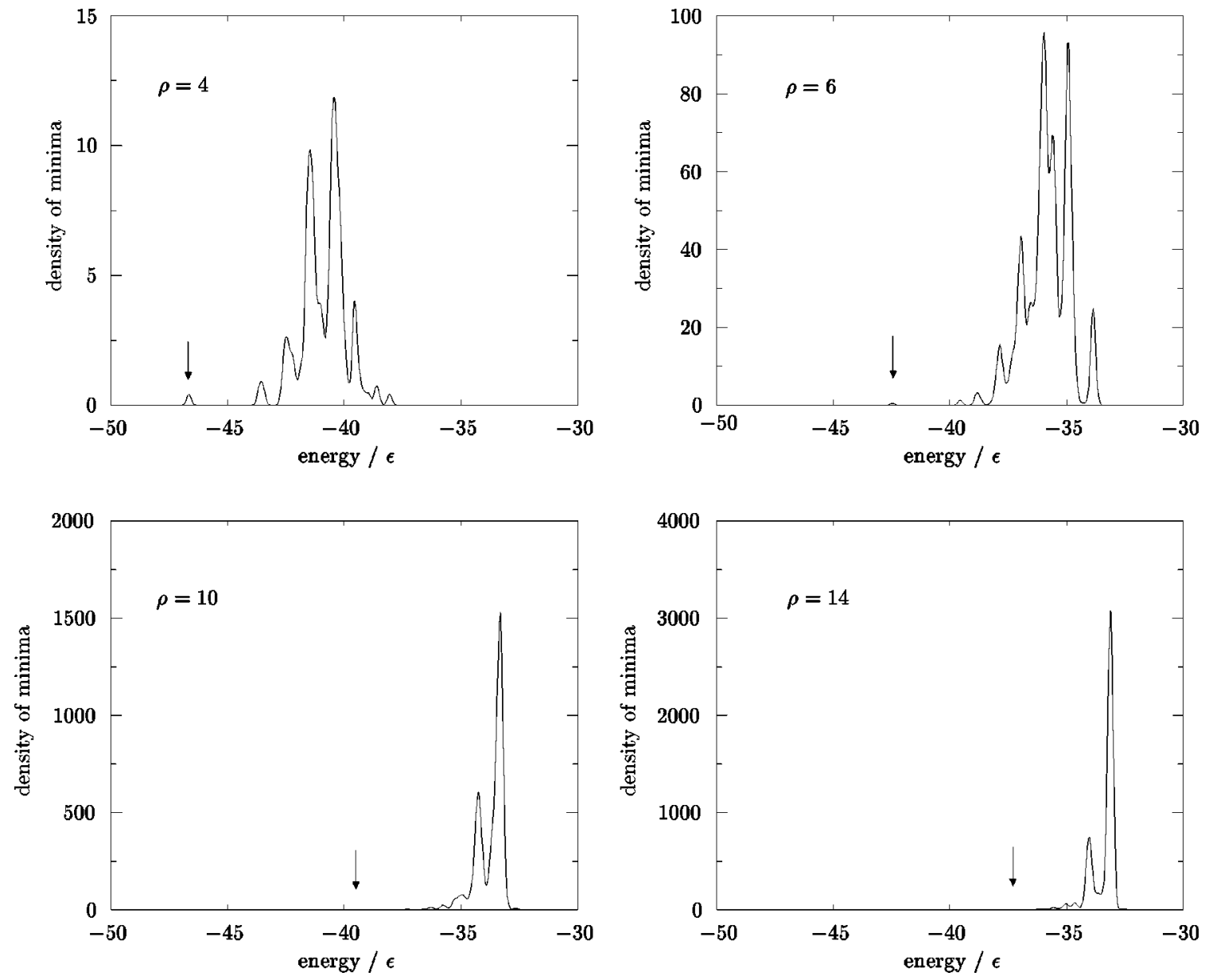

FIG. 4. Energy distribution of the minima for four values of the range parameter $\rho$. In each case, the energy of the global minimum is indicated by an arrow.

becomes the second lowest minimum. In fact, for $\rho$ $>14.77$, the decahedron is the global minimum, although this value of $\rho$ may be too large to be observed in chemical systems. The change in the order of the stationary point arises from a delicate balance between $E_{\text {strain }}$ and $E_{\mathrm{nnn}}$. The vibrational mode of the decahedron with the lowest Hessian eigenvalue is a twist about the $C_{5}$ axis. This motion strains the structure, but brings non-nearest-neighbors closer. At high $\rho$, the increased strain wins, causing the energy to rise and giving a minimum, whereas for longer-ranged interactions the non-nearest-neighbors lower the energy, giving a saddle.

The decreasing $\Delta E_{\text {gap }}$ indicates a local flattening of the PES at higher $\rho$. This effect extends beyond the vicinity of the global minimum to the whole landscape, as can be seen from the energy distributions of minima shown in Fig. 4. As $\rho$ increases, the energy distribution shifts upwards and becomes narrower, and for $\rho=10$ and 14, it develops two sharp peaks at -33 and -34 . At high values of $\rho, E_{\mathrm{nnn}}$ becomes small, and the energetic penalty for strain is large. Decomposition of the energy according to Eq. (2) reveals that the peaks in the distributions correspond to low-strain structures with 33 and 34 nearest neighbors. Low strain can arise from two structural motifs: close packing or polytetrahedral packing (without pentagonal bipyramids). It is not easy to classify such a small cluster according to these schemes, but it is worth noting that the radial distribution function, taken over all the minima, develops a $\sqrt{2}$ signature as $\rho$ increases, which is characteristic of close packing. ${ }^{38}$

A more quantitative measure of the slope of the PES is provided by the energy difference between pairs of connected minima, $\Delta E_{i}^{\mathrm{con}}$ (where $i$ labels the connecting transition state, or, equivalently the pathway). As Table II shows, the average of this quantity over the pathways drops off quickly as $\rho$ increases from $4 . \Delta E_{i}^{\text {con }}$ is the difference between the uphill and downhill barriers $b_{i}^{\text {up }}$ and $b_{i}^{\text {down }}$ defined by transition state $i$ and the two minima it connects. Although the average over the pathways of the uphill barrier, $\left\langle b^{\text {up }}\right\rangle_{\mathrm{p}}$, decreases as the range of the potential decreases, $\left\langle b^{\text {down }}\right\rangle_{\mathrm{p}}$ increases, i.e., the barriers that must be overcome for structural relaxation towards the global minimum are larger; the flattening of the funnel is accompanied by roughening.

Given the dramatic increase in the number of stationary points as the range of the potential decreases, and that the volume of accessible phase space will be reduced as the long range attraction is squeezed out, we must expect some change in the nature of the individual pathways between minima and their organization on the landscape. Defining $D_{i}$ as the separation in configuration space of the two minima connected by transition state $i$, Table II shows, as we might 
TABLE III. Distribution of the number of steps $n^{\text {gm }}$ lying on the shortest path from local minima to the global minimum at four values of the range parameter $\rho$.

\begin{tabular}{ccccc}
\hline \hline & \multicolumn{4}{c}{ Number of minima } \\
\cline { 2 - 5 }$n^{\mathrm{gm}}$ & $\rho=4$ & $\rho=6$ & $\rho=10$ & $\rho=14$ \\
\hline 1 & 105 & 188 & 71 & 148 \\
2 & 50 & 591 & 937 & 1116 \\
3 & 3 & 518 & 2887 & 3502 \\
4 & & 116 & 3315 & 4393 \\
5 & & 19 & 1644 & 2627 \\
6 & & 6 & 403 & 843 \\
7 & & & 47 & 120 \\
8 & & & 1 & 10 \\
\hline \hline
\end{tabular}

expect, that connected minima are on average closer when the potential is short ranged. This effect is accompanied by a decrease in the average of the integrated path length, $S_{i}$. It is interesting to see how the individual pathways are organized into routes to the global minimum. We have calculated the shortest path from each minimum to the global minimum, as measured by the total integrated path length $S_{i}^{\text {gm }}$ (the path with fewest steps between minima is generally longer). The average of $S_{i}^{\mathrm{gm}}$ is fairly insensitive to $\rho$, whilst the average of the number of steps along the corresponding pathways, $n_{i}^{\mathrm{gm}}$, increases. Thus, on average, the path for relaxation to the global minimum does not increase significantly in length, but becomes more rugged as more transition states must be crossed. Whereas every minimum at $\rho=4$ can reach the global minimum in either 1 or 2 steps, as many as 5 may be required at $\rho=14$. Table III shows how the minima are distributed over $n_{i}^{\mathrm{gm}}$, giving some insight into the connectivity of the landscape. The number of minima with $n_{i}^{\mathrm{gm}}=1$ tells us how many transition states are connected directly to the global minimum. The values are remarkably high, especially as permutational isomers are not included. Interestingly, the number of minima does not increase continuously as the sequences branch out from the global minimum (as one might expect in a funnel), but tails off quite gently.

An intuitive explanation for the constancy of $\left\langle S^{\mathrm{gm}}\right\rangle$ and the increase in $\left\langle n^{\mathrm{gm}}\right\rangle$ might be that paths are split into a larger number of subrearrangements. The number of atoms contributing to rearrangement $i$ can be measured by the cooperativity index $\widetilde{N}_{i}=N / \gamma_{i}$, where $\gamma_{i}$ is the moment ratio of displacement, which is defined by ${ }^{39}$

$$
\gamma_{i}=\frac{N \sum_{\alpha}^{N}\left|\mathbf{r}_{\alpha}(s)-\mathbf{r}_{\alpha}(t)\right|^{4}}{\left(\sum_{\alpha}^{N}\left|\mathbf{r}_{\alpha}(s)-\mathbf{r}_{\alpha}(t)\right|^{2}\right)^{2}},
$$

where $\mathbf{r}_{\alpha}$ is the Cartesian position vector of atom $\alpha$, and $s$ and $t$ denote the final and initial configurations in rearrangement pathway $i$. Table II shows that the average value of $\widetilde{N}_{i}$ is almost independent of $\rho$. In fact the distribution of $\widetilde{N}_{i}$ (from 1 to $N$ ) is remarkably similar for all four databases. This result contrasts with statistics previously obtained for the larger clusters $\mathrm{LJ}_{55}$ and $\left(\mathrm{C}_{60}\right)_{55}$, which showed that co-

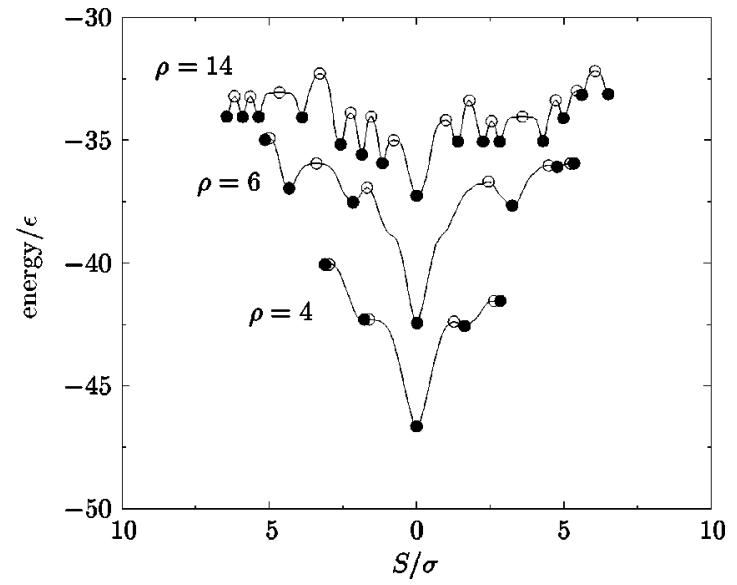

FIG. 5. Example monotonic sequences leading to the global minimum for three values of the range parameter $\rho . S$ is the integrated distance along the reaction path from the global minimum. Minima are indicated by filled circles, and the transition states by open circles. The plots demonstrate a number of features discussed in the text: the general increase in energy of the minima, the decreasing gap to the global minimum, the increasing barrier heights, the shorter rearrangements, and the decreasing gradient towards the global minimum as the range of the potential decreases.

operative (high $\tilde{N}_{i}$ ) rearrangements are less likely for $\left(\mathrm{C}_{60}\right)_{55}$, where the range of the potential corresponds to $\rho$ $\approx 14{ }^{29}$ It is possible that a 13 -atom cluster is too small to support localized subrearrangements in this way.

\section{SUMMARY}

We have performed a comprehensive survey of the potential energy landscapes of the 13-atom Morse cluster for four values of the range parameter using systematic eigenvector-following searches. The landscapes were then characterized in detail using disconnectivity graphs, funnel analysis, and a selection of parameters that provide insight into the topology and topography. We have described and rationalized the changes in the landscape as the range of the potential is varied over a physically meaningful range.

The trends displayed in Table II and the above discussion are underlined by the plots of representative monotonic sequences in Fig. 5. The overall classification of the potential energy landscape is that of a funnel, but one which becomes flatter and rougher as the range of the potential decreases. This change is accompanied by a general increase in complexity of the surface in terms of the number of minima and transition states and in the number of steps required to reach one minimum from another.

Previous studies of model potential landscapes ${ }^{6}$ have shown that relaxation from high-energy configurations to the global minimum is most efficient when the PES has a large potential energy gradient towards the global minimum with low downhill barriers, and lacks secondary funnels which act as kinetic traps. On this basis we would expect $\mathrm{M}_{13}$ to relax most easily when the the range of the potential is long, in spite of the fact that the frequency of intrawell vibrational oscillations decreases as the potential becomes less "stiff" at fixed values of $\epsilon$ and $r_{\mathrm{e}}$ (see Table II). Low values of the range parameter $\rho$ are therefore likely to produce "structure 
seekers," whereas high values will tend to produce "glass formers," reflecting a continuous change from a staircaselike to a sawtooth-like landscape.

Equipped with an understanding of the potential energy landscape and its dependence on the range of the potential, we have applied the master equation approach to investigate the dynamics of structural relaxation in $\mathrm{M}_{13}$. This work enables us to probe in detail the flow of probability between individual minima in an ensemble of clusters as they approach the equilibrium distribution, and the results will be described in a separate publication.

\section{ACKNOWLEDGMENTS}

M.A.M. and D.J.W. gratefully acknowledge financial support from the Engineering and Physical Sciences Research Council, and the Royal Society, respectively. M.A.M. would also like to thank Gonville \& Caius College, Cambridge for a grant towards attending the workshop on energy landscapes at the Telluride Summer Research Center in July 1997.

${ }^{1}$ J. D. Bryngelson, J. N. Onuchic, N. D. Socci, and P. G. Wolynes, Proteins: Struct., Funct., Genet. 21, 167 (1995).

${ }^{2}$ C. Levinthal, in Mössbauer Spectroscopy in Biological Systems, Proceedings of a Meeting Held at Allerton House, Monticello, Illinois, edited by P. DeBrunner, J. Tsibris, and E. Munck (University of Illinois Press, Urbana, 1969), p. 22.

${ }^{3}$ Ref. 2 is not readily available in libraries, but it can now be downloaded from URL http://brian.ch.cam.ac.uk/ mark/levinthal/levinthal.html.

${ }^{4}$ P. E. Leopold, M. Montal, and J. N. Onuchic, Proc. Natl. Acad. Sci. USA 89, 8721 (1992).

${ }^{5}$ P. G. Wolynes, Proc. Natl. Acad. Sci. USA 93, 14249 (1996).

${ }^{6}$ J. P. K. Doye and D. J. Wales, J. Chem. Phys. 105, 8428 (1996).

${ }^{7}$ A. Sali, E. Shakhnovich, and M. Karplus, J. Mol. Biol. 235, 1614 (1994).

${ }^{8}$ P. Amara and J. E. Straub, J. Phys. Chem. 99, 14840 (1995).
${ }^{9}$ C. A. Angell, J. Non-Cryst. Solids 131-133, 13 (1991).

${ }^{10}$ C. A. Angell, Science 267, 1924 (1995).

${ }^{11}$ F. H. Stillinger, Science 267, 1935 (1995).

${ }^{12}$ S. Sastry, P. G. Debenedetti, and F. H. Stillinger, Nature (London) 393, 554 (1998).

${ }^{13}$ R. E. Kunz and R. S. Berry, J. Chem. Phys. 103, 1904 (1995).

${ }^{14}$ K. D. Ball, R. S. Berry, R. E. Kunz, F.-Y. Li, A. Proykova, and D. J. Wales, Science 271, 963 (1996).

${ }^{15}$ F. H. Stillinger and T. A. Weber, Phys. Rev. A 25, 978 (1982).

${ }^{16}$ J. P. K. Doye and D. J. Wales, J. Phys. B 29, 4859 (1996).

${ }^{17}$ M. R. Hoare and J. McInnes, Faraday Discuss. 61, 12 (1976).

${ }^{18}$ P. A. Braier, R. S. Berry, and D. J. Wales, J. Chem. Phys. 93, 8745 (1990).

${ }^{19}$ J. P. K. Doye, D. J. Wales, and R. S. Berry, J. Chem. Phys. 103, 4234 (1995).

${ }^{20}$ J. P. K. Doye and D. J. Wales, J. Chem. Soc., Faraday Trans. 93, 4233 (1997).

${ }^{21}$ J. P. K. Doye and D. J. Wales, Science 271, 484 (1996).

${ }^{22}$ D. T. Mainz and R. S. Berry, Mol. Phys. 88, 709 (1996).

${ }^{23}$ P. M. Morse, Phys. Rev. 34, 57 (1929).

${ }^{24}$ L. A. Girifalco and V. G. Weizer, Phys. Rev. 114, 687 (1959).

${ }^{25}$ L. A. Girifalco, J. Phys. Chem. 96, 858 (1992).

${ }^{26}$ J. N. Murrell and K. J. Laidler, J. Chem. Soc., Faraday Trans. 64, 371 (1968).

${ }^{27}$ J. Pancír r, Collect. Czech. Chem. Commun. 40, 1112 (1974).

${ }^{28}$ C. J. Cerjan and W. H. Miller, J. Chem. Phys. 75, 2800 (1981).

${ }^{29}$ D. J. Wales, J. Chem. Phys. 101, 3750 (1994).

${ }^{30}$ M. Page and J. W. McIver, J. Chem. Phys. 88, 922 (1988).

${ }^{31}$ C. J. Tsai and K. D. Jordan, J. Phys. Chem. 97, 11227 (1993).

${ }^{32}$ O. M. Becker and M. Karplus, J. Chem. Phys. 106, 1495 (1997).

${ }^{33}$ P. Sibani, J. C. Schön, P. Salamon, and J. Andersson, Europhys. Lett. 22, 479 (1993).

${ }^{34}$ R. S. Berry and R. Breitengraser-Kunz, Phys. Rev. Lett. 74, 3951 (1995).

${ }^{35}$ J. Pillardy and L. Piela, J. Phys. Chem. 99, 11805 (1995).

${ }^{36}$ D. J. Wales, M. A. Miller, and T. R. Walsh, Nature (London) 394, 758 (1998).

${ }^{37}$ J. P. K. Doye and D. J. Wales, Chem. Phys. Lett. 247, 339 (1995).

${ }^{38}$ N. W. Ashcroft and N. D. Mermin, Solid State Physics (Holt, Rinehart and Winston, New York, 1976).

${ }^{39}$ F. H. Stillinger and T. A. Weber, Phys. Rev. A 28, 2408 (1983). 